\title{
Análisis del punto de equilibrio del efectivo de un sistema de producción bovina en el Departamento del Beni, Bolivia
}

\author{
Pinto, R.C. ${ }^{1}$ and Lerdon, F.J. ${ }^{\circledR}$
}

\author{
'Proyecto de Alianzas Rurales. Ministerio de Desarrollo Rural y Tierras. Santa Cruz. Bolivia. \\ ${ }^{2}$ Instituto de Economía Agraria. Universidad Austral de Chile. Valdivia. Chile.
}

\author{
Palabras ClaVe \\ Bovinos de carne. \\ Costos de producción. \\ Estudio de Caso. \\ Unidad de Producción Agropecuaria.
}

\section{RESUMEN}

El objetivo de este trabajo es analizar el Punto de Equilibrio del Efectivo (PEE) de un sistema de producción bovina de carne, en una Unidad de Producción Agropecuaria (UPA) del Departamento del Beni, Bolivia (Beni/Bo). La recolección de la información se hizo mediante visitas a terreno y el acompañamiento técnico a la gestión contable durante la temporada agrícola 2018-2019. Se utilizaron las técnicas de entrevistas abiertas, observación directa y registros en el cuaderno de campo. Las operaciones económicas, por su parte, fueron registradas en el software de Contabilidad de Gestión Agrícola (CONGA), disponible en el Instituto de Economía Agraria de la Universidad Austral de Chile. A partir de la información histórica generada por el software, se determinó las estructuras de costos e ingresos y se procedió a calcular el beneficio de la explotación y el PEE. Finalmente, se determinó la composición del hato ganadero que permite al productor alcanzar el PEE. Los resultados revelan que el Ingreso Bruto (B) anual resulta insuficiente para cubrir los Costos Totales (CT). Para cubrir los CT que generan una salida de efectivo, alcanzando un flujo de efectivo operativo de cero (PEE), el sistema productivo deberá producir $6.489 \mathrm{Kg}$ de carne Peso Gancho de res para la venta, comercializados a un precio de carne de res en gancho de $16 \mathrm{Bs} / \mathrm{Kg}$. Para que el sistema productivo arroje anualmente estos $6.489 \mathrm{Kg}$, deberá el hato ganadero contar mínimamente con 85 vacas reproductoras, manteniendo una relación toro vaca de $5 \%$, nacimiento de terneros $73 \%$, mortalidad de terneros $4 \%$ y bovinos adultos $2 \%$.

\section{Analysis of the equilibrium point of the cash of a bovine production system in the Department of Beni, Bolivia}

\section{SUMMARY}

\section{ADDITIONAL KEYWORDS}

Agricultural Production Unit.

Beef cattle.

Case study.

Production costs.

INFORMATION

Cronología del artículo.

Recibido/Received: 17.06.2020

Aceptado/Accepted: 10.12 .2020

On-line: 15.01 .2021

Correspondencia a los autores/Contact e-mail:

jlerdon@uach.cl
The objective of this paper is to analyze the Point of Equilibrium of the Cash (PEE) of a bovine meat production system, in an Agricultural Production Unit (UPA) of the Department of Beni, Bolivia. The collection of the information was done through field visits and the technical accompaniment to the accounting management during the agricultural season 2018-2019. The techniques of open interviews, direct observation and recording in the field notebook were used. The economic operations, on the other hand, were registered in the agricultural management accounting software called CONGA. From the historical information generated by the soffware, the cost and income structures were determined and proceeded to calculate the benefit of the operation and the PEE. Finally, the composition of the livestock herd that allows the producer to reach the PEE was determined. The results reveal that the annual Gross Income is insufficient to cover the Total Costs (TC). In order to cover the CT that generate a cash oufflow, reaching an operational cash flow of zero (cash balance point), the production system must produce $6.489 \mathrm{Kg}$ of meat Weight Hook of beef for sale, marketed at a price of beef on a hook of $16 \mathrm{Bs} / \mathrm{Kg}$. In order for the productive system to produce $6,489 \mathrm{~kg}$ annually, the herd must have at least 85 breeding cows, maintaining a bull-cow ratio of $5 \%$, birth of calves $73 \%$, mortality of calves $4 \%$ and adult cattle $2 \%$.

\section{INTRODUCCIÓN}

La ganadería desempeña un papel fundamental como motor del desarrollo del sector agrícola y alimentario, impulsora de importantes cambios económicos, sociales y ambientales en los sistemas alimentarios de todo el mundo, siendo fundamental para comprender las cuestiones que giran en torno al desarrollo agrícola sostenible (HLPE 2016, p. 133). Sin embargo, la gran diversidad de sistemas ganaderos en todo el mundo hace que esta tenga efectos variables en la viabilidad socioeconómica de las comunidades, en los recursos naturales y en el medio ambiente (HLPE 2016, p. 39).
En el Departamento del Beni/BO, la ganadería es una de las principales actividades económicas, responsable del 25\% del Producto Interno Bruto departamental (Fundación Milenio 2011, p. 1). Esta se ha especializado en la producción bovina de carne, bajo el sistema de cría y recría extensiva, basada en el aprovechamiento de sus extensas praderas naturales, que abarcan 5.8 millones de hectáreas, equivalentes al $65 \%$ del total de la superficie utilizada en actividades agropecuarias (INE n.d.). Actualmente, cuenta con una población que sobrepasa los 2.8 millones de bovinos, que representan el $32 \%$ del hato ganadero de Bolivia, número que lo 
sitúa, luego del Departamento de Santa Cruz, como el segundo Departamento con mayor número de bovinos en el país, habiendo decrecido en $12 \%$ en los últimos 10 años (INE n.d.a.).

Conforme a Aguilera (2004, pp. 2-3), en el Departamento del Beni existen 20.762 Unidades de Producción Agropecuaria (UPA) y aproximadamente 7.000 productores ganaderos, los cuales han sido clasificados, considerando el número de cabezas que poseen, en grandes, medianos, pequeños y familiares o de subsistencia (Tabla I). La mayoría (69\%) posee menos de 100 cabezas y pertenece a la categoría familiar. Le siguen los pequeños ganaderos que poseen entre 101 a 500 cabezas, los que representan el 17\% del total y producen fundamentalmente para el mercado local, con el empleo de fuerza de trabajo familiar y jornaleros temporales, aunque suelen contar con mano de obra asalariada. A continuación, se encuentran los medianos productores que tienen entre 501 a 2.500 cabezas y los grandes ganaderos que poseen más de 2.500 cabezas, representando el 11\% y $3 \%$ del total de los productores ganaderos del Beni respectivamente. Estos se caracterizan por poseer grandes extensiones de tierras y producir sobre la base de la compra de fuerza de trabajo asalariada permanente y temporal (Ormachea \& Ramírez 2011, p. 5).

Fuente: Elaboración en base a datos extraídos de Aguilera, 2004.

Respecto al sistema de explotación ganadera, en muchos casos este sigue siendo empírico y rudimen-

\begin{tabular}{cccc}
\hline $\begin{array}{l}\text { Tabla I. Clasificación de los productores ganaderos } \\
\text { del departamento del Beni (Classification of livestock pro- } \\
\text { ducers in the Beni department). }\end{array}$ \\
\begin{tabular}{cccc} 
Categoría & Nro. de Cabezas & $\begin{array}{c}\text { Nro. de Pro- } \\
\text { ductores }\end{array}$ & $\%$ \\
Grande & $>2.501$ & 228 & 3 \\
Mediano & 501 a 2.500 & 792 & 11 \\
Pequeño & 101 a 500 & 1.168 & 17 \\
Familiar & $<100$ & 4.867 & 69 \\
& Total & 7.055 & 100 \\
\hline
\end{tabular}
\end{tabular}

Fuente: Elaboración en base a datos extraídos de Aguilera, 2004.

tario. Sin embargo, un considerable número de empresas ganaderas ha pasado del sistema de cría extensiva tradicional a un sistema extensivo mejorado, con miras a una mayor productividad. Estos productores, en su mayoría están estandarizando sus hatos, porque no pueden crecer en número de bovinos, limitados por la extensión de tierra (Aguilera 2004, p. 4).

Un concepto relevante, en la determinación de la dimensión más adecuada de la empresa, es el denominado punto de equilibrio, técnica de análisis empleada como instrumento de planificación que puede usarse en la gestión de las empresas agropecuarias, y que permite determinar el nivel de Ingresos Brutos (IB) que deben ser generados para cubrir todos los costos de explotación (Helguera \& Lanfranco 2006, p. 13). Sin embargo, la gran mayoría de las explotaciones ganaderas en nuestro medio no realizan el registro de las diferentes operaciones económicas que ejecutan y menos bajo un sistema contable. Presentan información muchas veces incompleta que no permite realizar un análisis exhaustivo del punto de equilibrio.

Esta situación se debe a que, en la mayoría de los casos, la gestión de la explotación ganadera depende directamente del propietario, quien cuenta con conocimientos teóricos y prácticos sobre el manejo de animales y antecedentes del predio, pero no sobre contabilidad de gestión, que le permitan planificar, controlar y evaluar permanentemente su explotación. A su vez, las instituciones de asistencia técnica, investigación y transferencia tecnológica agropecuaria (público, mixto y privado), se han centrado principalmente en los aspectos relacionados al mejoramiento genético y de medidas de control sanitario.

Por otra parte, existen estudios que han estimado el costo de producción y la viabilidad económica de la producción de carne bovina en los variados sistemas de producción (Cezar \& Euclides Filho, 1996, pp. 5-31; Alves et al. 1999, pp. 1-4; Pötter et al. 2000, pp. 861870; Aguiar et al. 2001, pp. 1462-1464; Lerdon et al. 2015, p. 14; Lerdon et al. 2016, pp. 53-64). Sin embargo, no profundizan en los factores que influenciaron el costo, así como en los componentes que ejercieron mayor influencia. Debido a esta falta de información que asocie la producción con el costo de obtenerla en sistemas de producción de carne bovina, se hace necesario identificar los componentes del costo total y operativo efectivo, que tienen mayor representatividad en la producción del kilo de carne y estimar el punto de equilibrio del sistema de producción. El no conocer el punto de equilibrio puede traer como consecuencia que las empresas gasten más de lo que una situación económica y financiera sana permite, ocasionando problemas administrativos y financieros. Esta situación, sostenida por períodos relativamente largos, puede llevar al cierre de las empresas.

En este escenario, el objetivo de este estudio fue establecer el punto de equilibrio del efectivo de un sistema de producción bovina de carne, en una unidad de producción agropecuaria del Departamento del Beni en Bolivia, facilitando así información financiera que permita al productor, dedicado a esta actividad, determinar la dimensión más adecuada de su empresa ganadera.

\section{MATERIAL Y MÉTODOS}

\section{UBICACIÓN GEOGRÁFICA DE LA UPA}

Este estudio se realizó en una UPA localizada en la región Amazónica de Bolivia, específicamente en el municipio de Guayaramerín, situado en la provincia Antonio Vaca Diez, al norte del Departamento del Beni. El territorio de esta provincia está compuesto en su mayor parte por montes altos y campos de pastoreo. El clima local, según la clasificación de Köppen, es tropical húmedo del tipo Aw. Con una temperatura media de $26^{\circ} \mathrm{C}$ y una precipitación anual de 1.780 $\mathrm{mm}$, distribuidos en un periodo relativamente seco, de mayo a septiembre (precipitación mensual $<100 \mathrm{~mm}$ ) 
y un periodo lluvioso de octubre a abril (Beekma et al. 1996, p. 11).

\section{OBTENCIÓN Y ANÁLISIS DE LA INFORMACIÓN}

La recolección de información se realizó mediante visitas a terreno y el acompañamiento técnico a la gestión contable de la UPA estudiada durante la temporada agrícola 2018-2019. Se utilizaron las técnicas de entrevistas abiertas, observación directa y registro en el cuaderno de campo. Las operaciones económicas fueron recogidas en unidad de boliviano (Bs.) y registradas en el software de Contabilidad de Gestión Agrícola denominado CONGA, que es un conjunto de procedimientos computacionales y manuales diseñado por investigadores del Instituto de Economía Agraria de la Universidad Austral de Chile, el cual permite el registro, proceso de datos y obtención de información, tanto presupuestaria como contable, resultante de la explotación de empresas agropecuarias.

A partir de la información histórica generada por el software CONGA, se determinó, en hojas de cálculo del programa EXCEL, las estructuras de costos e ingresos antes de intereses, impuestos, depreciación y amortizaciones, y se procedió a calcular el beneficio de la explotación y el Punto de Equilibrio del Efectivo (PEE). El objetivo de este análisis consistió en determinar el nivel de ventas que resulta en un flujo de efectivo operativo de cero (Ross et al. 2014, p. 347). Es decir, se trató de encontrar el Ingreso Bruto (IB) en efectivo que una empresa necesita generar en el ejercicio de su actividad, para cubrir los costos que le generan una salida de efectivo durante un período determinado.

En la contabilidad agropecuaria, por lo general, el IB no solo proviene de la producción y comercialización de distintos bienes y servicios, sino también, de los ingresos que no reflejan una entrada de efectivo, como la producción de alimentos o semillas consumida en el predio, los productos empleados y recibidos en especie $\mathrm{y}$, fundamentalmente, los cambios en el valor de inventario por el desarrollo biológicos de los activos, como las plantas y animales vivos (Helguera \& Lanfranco 2006, p. 15).

En la actividad de cría y recría de bovinos, la parte del IB que genera una entrada de efectivo prácticamente proviene de la venta de animales en pie para faena, recría o engorda, así como de la venta de Charque. Por su parte, el nacimiento de los terneros/as y los cambios en el valor de inventario por el desarrollo biológico de los bovinos, así como el consumo patronal o la producción consumida durante el año agrícola, corresponden a la parte del IB que no genera una entrada de efectivo. Los Costos Totales (CT) por su parte, son la suma de los Costos Fijos (CF) y los Costos Variables (CV). Por definición, los CF son aquellos que no cambian cuando la cantidad de producción experimenta un cambio durante un periodo específico. Los CV, al contrario, se modifican cuando hay un cambio en el nivel de producción y llegan a cero cuando la producción es nula (Ross et al. 2014, p. 338).

Cuando en la producción se identifica claramente un producto principal, que es el que realiza el principal aporte al IB, resulta suficiente calcular el punto de equilibrio para éste único producto. De no ser ésta la situación, se deberá calcular el punto de equilibrio de cada producto y luego el global de la empresa (Helguera \& Lanfranco 2006a, p. 52). En este estudio se identificó, como producto principal, la "venta de animales en pie para faena", expresada en kilogramos, por ser este significativamente determinante del IB que genera una entrada de efectivo en el sistema productivo de cría y recría de la UPA estudiada. A continuación, se calculó el PEE con los ingresos y egresos que reflejan una entrada y salida de efectivo, considerando a $Q$ como el total de unidades vendidas en un determinado periodo; $\mathrm{CF}$, los costos fijos totales; $\mathrm{P}$, el precio unitario de venta y CVU, el costo variable por unidad de producto. Por tanto:

$$
\mathrm{PEE}=Q=\frac{\mathrm{CF}}{\mathrm{P}-\mathrm{CVU}}
$$

A la diferencia entre el precio y el costo variable por unidad de producción ( $P$ - CVU) se le denominó margen de contribución unitario (MCU). Este representa el excedente o margen bruto, luego de cubrir el CV de producir cada unidad de producto, para ir absorbiendo una parte cada vez mayor de CF (Helguera \& Lanfranco 2006, p. 17).

Finalmente, se determinó la composición del hato ganadero que permite al productor alcanzar el PEE. Para ello, se elaboró en una hoja de cálculo del programa EXCEL, la proyección del hato ganadero para el periodo de un año agrícola y calculó el número óptimo de vacas reproductoras que permitían al sistema productivo generar los animales necesarios para alcanzar dicho PEE. Para realizar lo anterior, se tomó como referencia los indicadores zootécnicos del sistema extensivo mejorado de cría y recría bovina del departamento del Beni, señalados por Aguilera (2004, p. 14), y los pesos promedio de venta de los animales en pie para faena registrados durante la temporada agrícola 2018-2019.

\section{RESULTADOS Y DISCUSIÓN}

\section{DESCRIPCIÓN DE LA UPA}

La UPA abordada en este estudio pertenece a la categoría de pequeña empresa ganadera. Esta presenta una superficie de 87 ha, de las cuales 79 ha son áreas de pastoreo, campos limpios con pasto introducido del género Brachiaria, destinados a la producción bovina de carne. Alrededor de 5 ha son utilizadas para el cultivo de yuca para autoconsumo bajo el sistema de roza, tumba y quema. El predio también presenta diversidad de árboles frutales: Carambola, Manga, Cacao, Limón, Lima, Naranja, destacando el Copoazú por tener mayor presencia; y animales menores: gallinas, patos, cerdos y ovejas en pequeña cantidad orientados también al autoconsumo.

La UPA no cuenta con energía eléctrica permanente, pero si con un generador de luz y acceso al agua (no potable) proveniente de pozos y arroyo. Cuenta también con infraestructura e instalaciones apropiadas: vivienda para el personal, corral y brete de manejo, 
divisiones de potreros, comederos, bebederos, porqueriza y gallinero para el manejo adecuado de los animales y el habitar del personal.

La mano de obra permanente se reduce a un capataz que vive en la UPA con toda su familia. Este, cuida a los animales y realiza tareas de mantenimiento del predio. La mano de obra ocasional la componen diaristas que auxilian al capataz y al productor, principalmente en el manejo del ganado bovino, rozas de pastizales, establecimiento del cultivo de yuca y otras tareas de apoyo para el mantenimiento del predio. El productor, reside en la sede del municipio de Guayaramerín, a 18 $\mathrm{Km}$ de la UPA, donde tiene otra actividad económica, realizando visitas semanales al predio.

\section{Características del SISTEMA DE PROdUCCIÓN BOVINA DE CARNE}

El proceso productivo está compuesto por las fases de cría y recría. El método de reproducción es la monta natural continua con ausencia de diagnósticos de preñez. El destete de los terneros/as también es natural al alcanzar aproximadamente los 10 meses de edad. La identificación de los bovinos es realizada a través de la marcación a fuego. No se practica la castración, ni el descorné de torillos, que son comercializados en pie para la faena al superar los tres años junto con las vacas de descarte, equivalentes al $20 \%$ de los vientres del sistema.

Semanalmente, el hato ganadero es sometido a un control sanitario donde se adoptan las siguientes medidas profilácticas: en los terneros recién nacidos, son retiradas las larvas del ombligo y se aplica un larvicida, vermicida, repelente y cicatrizante en aerosol, que también es utilizado sobre las lesiones de los bovinos producidas por cortes o cualquier otro tipo de herida. Para proteger al ganado de los ectoparásitos, principalmente de las garrapatas y mosca de cuernos, les son aplicadas periódicamente en el dorso de los bovinos, dosis de insecticidas y acaricidas de acción prolongada, adicionalmente es preparada una solución con creolina y aceite de motor usado, la cual es aplicada sobre las zonas habitadas por garrapatas en los bovinos hospedatarios. Sin embargo, la Piroplasmosis o Babesiosis (enfermedad transmitida por la garrapata) tiene una alta incidencia en el predio, ocasionando considerables pérdidas económicas. Para el control y tratamiento de esta infección parasitaria, en los bovinos infectados se aplica un hemoparasiticida de acción múltiple a base de Diminaceno, Oxitetraciclina y Dipirona. Además, es administrado hierro tipo III intravenoso diluido en suero fisiológico para tratar la anemia causada por esta enfermedad. Por otra parte, para complementar los requerimientos nutricionales y de vitaminas, es suministrado en los bovinos una solución inyectable con vitamina A, D y E. El control de la fiebre aftosa es realizado anualmente conforme campaña nacional de vacunación. Otros medicamentos que actúan como protector hepático, antitóxico y antibióticos, también suelen ser administrados de acuerdo a requerimiento de los animales.

Los pastos son el principal recurso renovable de la UPA y la base de la alimentación y fuente de nutrientes del hato ganadero. El área de pastoreo está dividida en 8 potreros irregulares manejados de forma diferida, que consiste en mantener durante el periodo de final de las lluvias, áreas y/o potreros sin animales, con la finalidad de acumular forraje para su utilización durante el periodo seco (Costa 2004, p. 25). Una época en la que los animales padecen de deficiencias minerales y el forraje, alimento del cual dependen exclusivamente, se encuentra en menor cantidad. La suplementación mineral, por su parte, es realizada con sal común durante la época de lluvia. En la época seca es ofertado un concentrado elaborado a base de maíz, jachi de almendra, suplementos minerales y sal común. En este sentido, el cuidado de la salud de los animales, además de la suministración de complementos alimenticios, son elementos fundamentales para mejorar la productividad y por tanto hacer rentable la actividad ganadera (Castillo, Kerguelen \& Negrete 2015, p. 133).

El sistema así conducido, como se aprecia en la Tabla II, presenta una tasa de nacimientos del $47 \%$, que es menor al promedio registrado en los sistemas extensivos tradicional y mejorado del departamento del Beni, señalados por Aguilera (2004, p. 14). Lo anterior, puede explicarse por la incorporación tardía de vacas de reemplazo al proceso de reproducción a partir de una crianza inadecuada (Ríos \& Benítez 2015, p. 414). Por otra parte, se observa una elevada mortalidad de terneros / as durante la crianza y de bovinos adultos durante la recría, equivalentes al 20\% y $11 \%$ respectivamente, que impide una adecuada reposición y mantenimiento del hato ganadero. En cuanto a la tasa de extracción, esta es del 12\% resultando también inferior al promedio registrado en los sistemas extensivos tradicional y mejorado del departamento.

\section{COMPOSICIÓN DEL HATO GANADERO}

El rebaño de la UPA está constituido por bovinos de la raza mestiza Nelore, que acorde a Ikeda et al.

Tabla II. Índices productivos según sistema de cría y recría bovina en el departamento del Beni (Production rates according to bovine breeding and breeding system in the Beni department).

\begin{tabular}{|c|c|c|c|}
\hline Índice & $\begin{array}{c}\text { Sistema } \\
\text { UPA estu- } \\
\text { diada }\end{array}$ & $\begin{array}{l}\text { Siste m a } \\
\text { Extensivo } \\
\text { Tradicional }\end{array}$ & $\begin{array}{l}\text { Sistema Ex- } \\
\text { tensivo } \\
\text { Mejorado }\end{array}$ \\
\hline Nacimientos & $47 \%$ & $52 \%$ & $73 \%$ \\
\hline $\begin{array}{l}\text { Mortalidad de } \\
\text { terneros/as }\end{array}$ & $20 \%$ & $7 \%$ & $4 \%$ \\
\hline $\begin{array}{l}\text { Mortalidad } \\
\text { bovinos adultos }\end{array}$ & $11 \%$ & $4 \%$ & $2 \%$ \\
\hline Extracción & $12 \%$ & 15 & $16 \%$ \\
\hline
\end{tabular}


Tabla III. Masa ganadera de la UPA (UPA livestock mass).

\begin{tabular}{lcccc}
\hline $\begin{array}{l}\text { Categoría } \\
\text { Bovino }\end{array}$ & $\begin{array}{c}\text { UA } \\
\text { equivalente }\end{array}$ & $\begin{array}{c}\text { Número de } \\
\text { males }\end{array}$ & $\begin{array}{c}\text { ani- } \\
\text { Cabezas }\end{array}$ & UA \\
$\begin{array}{l}\text { Toros Repro- } \\
\text { ductores }\end{array}$ & 1,5 & 4 & 6 & 7,0 \\
Vacas & 1,0 & 50 & 50 & 58,1 \\
Terneros & 0,1 & 5 & 0,5 & 0,6 \\
Terneras & 0,1 & 5 & 0,5 & 0,6 \\
Torillos 1 año & 0,4 & 5 & 2 & 2,3 \\
Vaquillas 1 año & 0,3 & 12 & 4 & 4,7 \\
Torillos 2 años & 0,7 & 11 & 8 & 9,3 \\
Vaquillas 2 & 0,7 & 15 & 11 & 12,8 \\
años & 1,2 & 3 & 4 & 4,7 \\
Torillos 3 años & & 110 & 86 & 100 \\
Totales & & &
\end{tabular}

Fuente: Elaboración en base a fuentes primarias.

(2019, p. 78) es la de mayor importancia entre las razas de producción de carne en Bolivia. En la Tabla III se puede observar que el 58,1\% del total de hato ganadero, expresados en Unidad Animal (UA), lo constituyen las vacas reproductoras, $17,5 \%$ vaquillas de uno a dos años que son utilizadas para la reposición, 7,0\% toros reproductores y 1,2\% terneros/as. El restante 16,3\% del hato ganadero lo conforman los torillos de 1 a 3 años.

\section{CAPACIDAD DE CARGA ANIMAL}

A la relación numérica entre la cantidad de animales que pastorean una superficie y la disponibilidad de alimento que esa superficie puede proporcionar, sin afectar su productividad de forraje, se la denomina capacidad de carga animal. Para Santos et al. (2011, p. 336) no existe una regla fija para la estimación de la capacidad de carga, esta debe ser flexible y espacialmente variable, dependiente de la proporción de los paisajes y de las condiciones climáticas. Por lo que cada productor deberá evaluar y validar el manejo adoptado en cada gestión haciendo los ajustes necesarios.

Conforme al Centro Nacional de Mejoramiento de Ganado Bovino del Beni (2010), la capacidad de carga en las pampas benianas ocupada con pastos nativos es de: 0,3 a $0,5 \mathrm{UA} / \mathrm{ha} /$ año frente a 1,0 a 1,3 UA/ha/año en pastos cultivados o introducidos. En la UPA estudiada la carga animal es 1,1 UA/ha/año, que resulta en su formulación más sencilla de dividir el total bovinos presentes (86 UA) entre el total de la superficie destinada al pastoreo (79 ha). Considerando estos datos, se puede establecer que la capacidad máxima de carga animal de la UPA es de 103 UA.

\section{ESTRUCTURA DE INGRESOS}

El Ingreso Bruto anual fue de Bs 132.287 y solamente el 25,1\% del IB genera una entrada de efectivo, estando compuesto por la venta de Charque $(1,6 \%)$ y de bovinos en pie para faena, tres torillos mayores a tres años $(6,7 \%)$ y diez vacas de descarte $(16,8 \%)$.
Por otra parte, el 74,9\% del IB que no refleja una entrada de efectivo está compuesto por la producción consumida $(1,6 \%)$, la producción anual de forraje que es obtenido de los potreros $(2,9 \%)$, los ingresos por los cambios en el valor del inventario provocado por el nacimiento de los terneros $(5,0 \%)$ y principalmente por el desarrollo biológico de los bovinos durante el año agrícola, que generan un cambio de categoría por edad y es registrado contablemente al final de la temporada como ventas internas de vaquillas $(36,6 \%)$ y torillos $(28,8 \%)$ de 1,2 y 3 años respectivamente.

\section{ESTRUCTURA DE COSTOS}

El Costo Total anual fue de Bs 158.775 el 54,9\% de esté no refleja una salida de efectivo, pero si cambios en el valor del inventario, generado esencialmente por el costo de reposición de animales $(39,6 \%)$ tras la venta interna de vaquillas y torillos de 1, 2 y 3 años al final de la temporada, seguido por los costos correspondientes a la previsión por pérdida por la mortalidad de los animales $(14,0 \%)$ y el retiro patronal $(1,3 \%)$ por el consumo de Charque.

Por otra parte, el 45,1\% del CT que genera una salida de efectivo, está compuesto por el costo de personal $(25,8 \%)$ por el pago mensual de salario y aguinaldo al personal permanente, el pago de jornales o diarias canceladas a personal eventual y el gasto en el alimento del personal. Seguido por los egresos incurridos en el alimento para el ganado $(7,9 \%)$, el cual incluye a los insumos utilizados en la elaboración de concentrados y la suplementación mineral de los bovinos, el maíz ofertado a los animales menores y el costo de pastoreo (que equivale al ingreso obtenido por la producción anual de forraje, mismo que no genera una salida de efectivo). Aparece a continuación el costo efectuado en productos veterinarios $(3,8 \%)$, el cual precede a los costos incurridos en la compra de combustibles y lubricantes (3,1\%), mantención y reparación de instalaciones, vehículo, maquinarias y equipos $(3,0 \%)$, honorarios profesionales por servicios veterinarios $(0,8 \%)$, costos diversos $(0,3 \%)$ y gastos de comercialización $(0,4 \%)$ referido al flete por el traslado de los bovinos al matadero, el aporte a la Asociación de Ganaderos por res faenada y la documentación sanitaria "Guía de Movimiento Animal", otorgada por el Servicio Nacional de Sanidad Agropecuaria e Inocuidad Alimentaria (SENASAG).

\section{COSTOS FIJOS}

El Costo Fijo (CF) anual fue de Bs 52.320 equivalente al 33,0\% del CT, que incluye el costo del personal $(25,8 \%)$ y el gasto realizado en la mantención y reparaciones de instalaciones, vehículo, maquinarias y equipos $(3,0 \%)$, que se anteponen a los gastos incurridos en combustibles y lubricantes $(3,1 \%)$, honorarios profesionales $(0,8 \%)$ y costos diversos $(0,3 \%)$.

\section{COSTOS VARIABLES}

El Costo Variable (CV) anual fue de Bs 106.455, equivalente al 67,0\% del CT. Esté incluye el costo por la reposición de animales $(39,6 \%)$ correspondiente a la mayor parte del $\mathrm{CV}$, seguido por el costo de previsión por la mortalidad de los animales $(14,0 \%)$, el cual se antepone a los gastos incurridos en el alimento para el ganado 
Tabla IV. Ingreso Bruto por la venta de animales en pie para faena (Gross income from the sale of standing animals for work).

\begin{tabular}{llll}
\hline Detalle & Cabezas & PG Kg & Bs \\
Venta Torillos $>3$ años & 3 & 555 & 8.880 \\
Venta Vacas de Descarte & 10 & 1.390 & 22.240 \\
Total & 13 & 1.945 & 31.120 \\
\hline
\end{tabular}

Fuente: Elaboración en base a fuentes primarias extraídas del software CONGA.

$(7,9 \%)$, productos veterinarios $(3,8 \%)$, retiro patronal $(1,3 \%)$ y el costo de comercialización $(0,4 \%)$.

\section{BENEFICIO DE LA EXPLOTACIÓN}

En este escenario el IB anual generado de Bs 132.287, resulta insuficiente para cubrir el CT de Bs 158.775. Por tanto, el presente sistema no es capaz de cubrir en su totalidad los CF y CV incurridos.

En general, se observa un sistema productivo de cría y recría extensivo de bovinos con algunas mejoras referentes al manejo sanitario y alimentación. Pero con una alta mortalidad de animales, que reduce la rentabilidad. Sin embargo, los resultados económicos desfavorables presentados por la UPA, deben considerarse teniendo en cuenta que el productor no vive exclusivamente de la ganadería, obteniendo otros ingresos que propician cierto alivio financiero y le permiten mantenerse en la actividad a la espera de una mejora en la productividad del sistema de cría y recría de bovinos anteriormente descrito.

\section{ANÁLISIS PUNTO DE EQUILIBRIO DEL EFECTIVO}

El cálculo del PEE, como se definió en la metodología, fue realizado para un único producto "venta de animales en pie para faena" expresada en kilogramos, por ser este significativamente determinante del IB que genera una entrada de efectivo. En la Tabla IV se observa que el ingreso por la venta de este producto es igual a Bs 31.120 y está compuesto por la venta de tres torillos mayores a 3 años y diez vacas de descarte con pesos promedio en pie para faena de 370 y $278 \mathrm{Kg}$ respectivamente, resultando en un peso Gancho (PG) total de $1.945 \mathrm{Kg}$, comercializados a un precio de carne de res en gancho de $16 \mathrm{Bs} / \mathrm{Kg}$.

El CF total que genera una salida de efectivo, es igual a Bs 52.320 y lo componen los gastos incurridos en personal Bs 40.930, honorarios profesionales Bs 1.200, mantención y reparaciones Bs. 4.789, combustible y lubricantes Bs 4.986 y finalmente los costos diversos Bs 415 . Por su parte, el CV total que refleja una salida de efectivo es igual a Bs 15.438 y agrupa los costos incurridos en alimentos para el ganado (sal, maíz, jachi de almendra y suplementos minerales) Bs 8.816 , productos veterinarios Bs 6.062 y la comercialización de los bovinos Bs 560 .

Atendiendo estos antecedentes, tanto la proporción de los CF como de los CV que significan una salida en efectivo para la UPA, coinciden con los expuestos en los estudios desarrollados por Ríos \& Benítez (2015, pp. 409-416) y Araujo et al. (2012, p. 86). En dónde la mano de obra y la alimentación de los animales corresponden

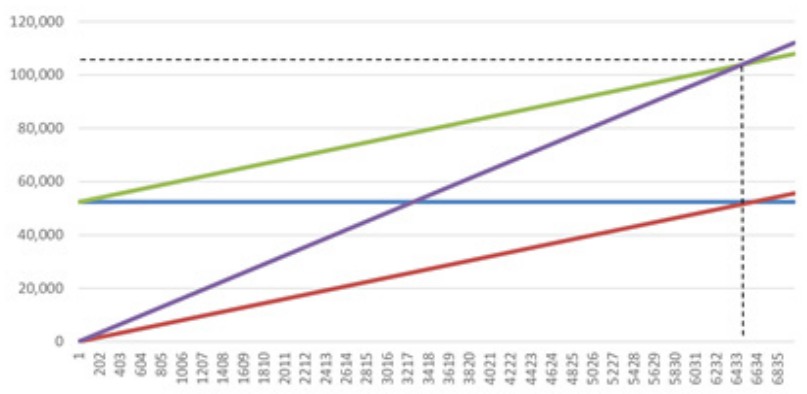

Figura 1.Punto de equilibrio del efectivo (Cash balance point).

a los principales costos que deben incurrir los ganaderos cárnicos de la Amazonía ecuatoriana y del Pantanal Matogrossense brasileño respectivamente, en sistemas de producción bovina similares.

A partir de lo expuesto, se obtiene el PEE que revela que la UPA estudiada deberá producir y vender 6.489 Kg de carne de res PG para cubrir los costos que le generan una salida de efectivo.

En la Figura 1, se observa que el PEE aparece representado por la intersección entre las curvas IB y CT. Nótese que, hasta que la UPA no alcance ingresos equivalentes a Bs 103.824 por la producción y comercialización de $6.489 \mathrm{Kg}$ de carne de res PG, a un precio de carne de res en gancho de $16 \mathrm{Bs} / \mathrm{Kg}$, se encontrará en la zona de pérdidas dado que no puede cubrir la totalidad de los costos que le generan una salida de efectivo.

\section{COMPOSICIÓN DEL HATO GANADERO QUE PERMITE ALCANZAR EL PUNTO DE EQUILIBRIO}

En la Tabla V se observa que es posible obtener 6.491 $\mathrm{Kg}$ de carne de res PG, que resultan suficientes para alcanzar el PEE de $6.489 \mathrm{Kg}$ anteriormente determinado. Con la venta de 40 bovinos, compuesto por 17 vacas y 23 torillos $>3$ años, resultantes del descarte del $20 \%$ de las vacas reproductoras y del total de los torillos mayores a 3 años.

Nótese también que para que el sistema productivo arroje anualmente este número de bovinos para la venta, el hato ganadero deberá contar con 85 vacas reproductoras, vigilando la relación toro vaca en alrededor del $5 \%$ y tomar acciones para mejorar sus índices de producción referentes al nacimiento de terneros/as y a la mortalidad de bovinos adultos y terneros/as. En efecto, mantener la mayor cantidad de vacas adultas en el rodeo conforme Ikeda et al. (2019, p. 84) posibilitaría desde un concepto zootécnico que los rasgos asociados a la eficiencia biológica como la longevidad y la reproducción de los animales produzcan una mayor sustentabilidad del sistema productivo. En esta línea, cabe destacar que tal como algunas investigaciones lo indican, las unidades de producción que cuentan con una mayor cantidad de vientres en producción generan niveles de excedentes superiores considerando inversiones más altas (Callejas et al. 2014, p. 138).

El manejo reproductivo tiene gran influencia en la eficiencia y rentabilidad del sistema de producción para obtener una eficiente productividad (Neto et al. 2018, 
Tabla V. Proyección del hato ganadero que permite alcanzar el punto de equilibrio (Projection of the livestock herd that allows to reach the break-equilibrium point).

\begin{tabular}{|c|c|c|c|c|c|c|c|c|c|c|}
\hline \multirow{3}{*}{ Categoría } & \multicolumn{2}{|c|}{ Cantidad } & \multicolumn{2}{|c|}{$(-)$} & \multirow{2}{*}{$\begin{array}{c}(-) \\
\text { Muertes }\end{array}$} & \multirow{2}{*}{$\begin{array}{c}(+) \\
\text { Nacimientos }\end{array}$} & \multirow{2}{*}{$\begin{array}{c}(-) \\
\text { Venta Int. }\end{array}$} & \multirow{2}{*}{$\begin{array}{c}(+) \\
\text { Compra Int. }\end{array}$} & \multicolumn{2}{|c|}{ (=) Cantidad } \\
\hline & $01 \mathrm{de}$ & Ene. & & nta & & & & & & Dic. \\
\hline & Cab. & UA & Cab. & $\mathrm{Kg}$. & Cab. & Cab. & Cab. & Cab. & Cab. & UA \\
\hline Toros & 5 & 7 & & & 1 & & & & 4 & 6 \\
\hline Vacas & 85 & 85 & 17 & 2.363 & 2 & & & 22 & 88 & 88 \\
\hline Terneros & 25 & 3 & & & 1 & 25 & 24 & & 25 & 3 \\
\hline Terneras & 25 & 3 & & & 1 & 25 & 24 & & 25 & 3 \\
\hline Torillos 1 año & 24 & 9 & & & 1 & & 23 & 24 & 24 & 9 \\
\hline Vaquillas 1 año & 24 & 8 & & & 1 & & 23 & 24 & 24 & 8 \\
\hline Torillos 2 años & 23 & 17 & & & 1 & & 22 & 23 & 23 & 17 \\
\hline Vaquillas 2 años & 23 & 16 & & & 1 & & 22 & 23 & 23 & 17 \\
\hline Torillo > 3 años & 23 & 27 & 23 & 4.128 & & & & 22 & 22 & 26 \\
\hline Totales & 257 & 175 & 40 & 6.491 & 9 & 50 & 138 & 138 & 258 & 177 \\
\hline
\end{tabular}

Fuente: Elaboración propia.

p. 52), debiendo ser atendidas también algunas de las principales barreras que coinciden con las que comúnmente afectan a los productores de carne, tales como los sistemas y manejo de pastoreos, conservación de forrajes, gestión de la UPA y el control sanitario de los animales (Rodríguez et al. 2018, p. 470). De esta manera, se contará con un hato ganadero relativamente estable compuesto por 257 bovinos y 175 UA.

\section{CONCLUSIONES}

Este estudio proporciono un parámetro de ingresos, costos, beneficio y del PEE de un sistema de producción bovina de carne, los cuales representan datos importantes para determinar la dimensión más adecuada de la empresa ganadera e implementar nuevas acciones en materia de gestión.

La producción animal en pie para faena, en este tipo de sistema de producción, no alcanza el PEE necesario para estabilizar financieramente la empresa ganadera. Los $1.945 \mathrm{Kg}$ de carne de res PG producidos y comercializados no cubre los costos totales de producción que generan una salida de efectivo. Para alcanzar el PEE es necesario aumentar la producción y venta de carne a $6.489 \mathrm{Kg}$ de carne de res PG.

Inversiones en vientres, capital de operación y mayor control sanitario principalmente, pueden ser alternativas para aumentar los índices productivos que le permitan al sistema productivo de cría y recría de bovinos arrojar anualmente 40 animales para la venta y así alcanzar el PPE. Sin embargo, si bien la capacidad de carga del predio permite al productor incrementar la cantidad de bovinos hasta 103 UA, esta resulta insuficiente para acoger a las 175 UA que mínimamente requiere el sistema productivo para estar en PEE.

\section{BIBLIOGRAFIA}

Aguiar, APA, Almeida, B, Amaral, GC, Datena, JLF, Younes, RJ, Costa, RO, Mota, J \& Vivan, WSO 2001, 'Viabilidade económica da produção de carne em sistemas intensivos de pastagens na região do cerrado',
In: 38a Reunião Anual da Sociedade Brasileira de Zootecnia, Piracicaba, pp. 1462-1464.

Aguilera, R 2004, La ganadería beniana en cifras, Federación de Ganaderos de Beni y Pando. Beni, Bolivia.

Alves, JB, Tarsitano, MAA, Bergamaschine, AF \& Bastos, JFP 1999, 'Desempenho econômico do confinamento de bovinos de diferentes grupos genéticos', In: 36a Reunião Anual da Sociedade Brasileira de Zootecnia, Porto Alegre, pp. 1-4.

Araujo, HS, Sabbag OJ, Lima, BTM, Andrighetto C, Ruiz U 2012, 'Aspectos econômicos da produção de bovinos de corte', Pesquisa Agropecuária Tropical, vol. 2, no. 1, pp. 82-89.

Beekma, J, Zonta, A \& Keiizer, B 1996. Base ambiental para el desarrollo Departamento de Pando y la Provincia Vaca Diez, SNV. La Paz, Bolivia.

Callejas, N, Aranda, H, Rebollar, S \& De La Fuente, L 2014, 'Situación económica de la producción de bovinos de carne en el estado de Chihuahua, México' Agronomía Mesoamericana, vol. 25, no. 1, pp. 133-139.

Castillo, O, Kerguelen, M \& Negrete, M 2015, 'Microeconomía de la producción de ganado vacuno de carne en el valle medio del rio Sinú (Montería - Colombia): un estudio de caso', Revista Facultad de Ciencias Económicas: Investigación y Reflexión, vol. 23, no. 2, pp. 123-135.

Ormachea, E \& Ramírez, N 2011, 'Producción de carne bovina en Bolivia y seguridad alimentaria', Boletín de seguimiento a políticas públicas, Centro de Estudios para el Desarrollo Laboral y Agrario, La Paz, septiembre.

Cezar, IM \& Euclides Filho, K. 1996, Novilho precoce: reflexos na eficiência e economicidade do sistema de produção. Campo Grande, EMBRAPA-CNPGC, Campo Grande, Brasil.

Centro Nacional de Mejoramiento de Ganado Bovino del Beni 2010, La ganadería del Beni y la calidad de los pastos nativos frente a la de los pastos introducidos, artículo elaborado por C Montaño, Centro Nacional de Mejoramiento de Ganado Bovino del Beni, Trinidad, Bolivia.

Costa, N 2004. Formação, manejo e recuperação de pastagens em Rondônia. Embrapa Rondônia, Porto Velho.

Fundación Milenio 2011, 'El Beni y su economía', Informe nacional de coyuntura, no. 124, Fundación Milenio, La Paz, 18 Noviembre.

Helguera, L \& Lanfranco, B 2006, Riesgo y rentabilidad en empresas ganaderas, Instituto Nacional de Investigación Agropecuaria, Montevideo, Uruguay.

Helguera, L \& Lanfranco, B 2006a, 'Análisis del punto de equilibrio empresarial', Revista del Plan Agropecuario, no. 117, pp. 50-55. 
HLPE 2016, Desarrollo agrícola sostenible para la seguridad alimentaria y la nutrición: ¿qué función desempeña la ganadería? Un informe del Grupo de alto nivel de expertos en seguridad alimentaria y nutrición del Comité de Seguridad Alimentaria Mundial, High Level Panel of Experts on Food Security and Nutrition, Roma.

Ikeda, A, Garzón, JP, Rodríguez, E \& Marini, P 2019, 'Comportamiento productivo y reproductivo de vacas nelore de diferentes edades en un sistema de pastoreo del trópico boliviano', Revista de las Agrociencias, no. 21 , pp. 77-86.

INE n.d., Ficha resúmen censo nacional agropecuario 2013, Instituto Nacional de Estadística, visto 18 septiembre 2018, <http://sice.ine. gob.bo/censofichacna>.

INE n.d.a, Ganadería, producción de carne y otros subproductos de las principales especies ganaderas y avícolas, Instituto Nacional de Estadística, visto 19 septiembre 2018, <https://www.ine.gob. bo/index.php/estadisticas-por-actividad-economica/ganaderiaproduccion-de-carne-y-otros-subproductos $>$.

Lerdon, J, Bentdjerodt, D, Carrillo, B \& Moreira V 2015, 'Análisis económico de 11 predios productores de leche y carne en la Región de Los Ríos, Chile', Revista IDESIA, vol. 33, no. 4, p. 14.

Lerdon, J, Herrera, I, Moreira, V \& Carrillo, B 2016. 'Análisis económico de predios ganaderos y lecheros del sur de Chile', Revista IDESIA, vol. 34 , no. 5, pp. 53-64.
Neto, AC, Viera, GHS, Haddade, IR, Rosado, TL \& Mello BLB 2018, 'Aplicação de novas tecnologias na bovinocultura leiteira', Revista Incaper, vol. 9, pp. 51-65.

Pötter, L, Lobato, J \& Netto, C 2000, 'Análises económicas de modelos de produção com novilhas de corte primíparas aos dois, três e quatro anos de idade', Revista da Sociedade Brasileira de Zootecnia, vol. 29, no. 3, pp. 861-870.

Ríos, S \& Benítez, D 2015, 'Análisis del funcionamiento económico productivo de los sistemas de producción cárnica bovina en la Amazonía Ecuatoriana', Archivos de. Zootecnia, vol. 64, no. 248, pp. 409-416. Rodríguez, S, Flores, D, León, A, Pérez, L, \& Aguilar, J 2018, 'Diagnóstico de sistemas de producción de bovinos para carne en Tejupilco, Estado de México', Revista Mexicana de Ciencias Agrícolas, vol. 9, no. 2, pp. 465-471.

Ross, S, Westerfield, R \& Jordan, B, trad. Valdés, MG 2014, Fundamentos de finanzas corporativas, McGraw-Hill, México D.F.

Santos, SA, Desbiez, AL, Crispim, SMA, Comastri Filho, JA, Abreu, UGP \& Rodela, LG 2011, Natural and cultivated pastures and their use by cattle', The Pantanal: ecology, biodiversity and sustainable management of a large neotropical seasonal wetland, Pensoft Publisher, Sofia, Bulgaria. 